\title{
The significance of exosomes in the development and treatment of hepatocellular carcinoma
}

\author{
Xin $\mathrm{Li}^{1}$, Chuanyun $\mathrm{Li}^{1}$, Liping Zhang ${ }^{3}$, Min $\mathrm{Wu}^{1}$, Ke Cao ${ }^{1}$, Feifei Jiang ${ }^{1}$, Dexi Chen ${ }^{2}$, Ning $\mathrm{Li}^{1,2^{*}}$ and Weihua $\mathrm{Li}^{2^{*}}$ (D)
}

\begin{abstract}
Hepatocellular carcinoma (HCC) is the most commonmalignancy. Exsome plays a significant role in the elucidation of signal transduction pathways between hepatoma cells, angiogenesis and early diagnosis of HCC. Exosomes are small vesicular structures that mediate interaction between different types of cells, and contain a variety of components (including DNA, RNA, and proteins). Numerous studies have shown that these substances in exosomes are involved in growth, metastasis and angiogenesis in liver cancer, and then inhibited the growth of liver cancer by blocking the signaling pathway of liver cancer cells. In addition, the exosomal substances could also be used as markers for screening early liver cancer. In this review, we summarized to reveal the significance of exosomes in the occurrence, development, diagnosis and treatment of HCC, which in turn might help us to further elucidate the mechanism of exosomes in HCC, and promote the use of exosomes in the clinical diagnosis and treatment of HCC.
\end{abstract}

\section{Key points}

- Liver is a multicellular organ that requires intercellular communication for implementing its function.

- The function of exosomes in intercellular communication has been acknowledged.

- During the formation of HCC, the tumor cells communicate with all kinds of liver cells to promote growth of $\mathrm{HCC}$ and metastasis, exerting a huge effect on exosomes.

- Because of the differences in HCC stage, different biomarkers are found in exosomes. Also exosomes can be used as carriers to release several substances. Some of these can regulate signal transduction pathways between HCC cells, and others can be used as drugs because of external membrane protection.

\footnotetext{
* Correspondence: liningya@ccmu.cn.edu; liweihua@163.com

${ }^{1}$ Beijing Youan Hospital, Capital Medical University, Beijing, China

${ }^{2}$ Beijing Institute of Hepatology, Beijing Youan Hospital, Capital Medical

University, 8 Xitoutiao, Youanmenwai,Fengtai District, Beijing 100069, China

Full list of author information is available at the end of the article
}

\section{Background}

Hepatocellular carcinoma (HCC) is the third most common malignancy in the world, accounting for $85-90 \%$ of primary liver cancer [1-3]. The annual incidence of new cases with HCC is estimated to be about 841,000 [4]. Hepatitis $B$ virus (HBV) infection is considered as the main risk factor for HCC development in China [5]. Despite new breakthroughs in imaging technology, chemotherapy, interventional radiology, surgical techniques, and liver transplantation in the recent years, the prognostic rate of patients with advanced liver cancer still remained poor, and there is no effective treatment method till date for it [6-8]. Currently, the 5-year survival rate of HCC is not more than $20 \%[9,10]$ and early diagnosis can significantly reduce the mortality in patients with HCC [11]. Therefore, the major methods to improve the total survival rate of HCC include improvementof the early diagnostic rate and explore the detailed formation mechanisms of HCC.

Exosomes are small nano-sized vesicles that transport biologically active molecules between cells, regulate microenvironment and immune system between cells by a variety of biomolecules (such as proteins, RNA and DNA) $[4,12,13]$, and the genetic and epigenetic mechanisms of cells [14]. Studies have shown that exosomes 
not only initiates the downstream signals to target cells, but also transfers the genetic material to downstream cells, providing intercellular communication with a new mechanism [4]. It has been reported that different types of exosomal compositions provided by databases such as Vesiclepedia, EVpedia, and Exocarta were found in different cells with similar physiological or pathological conditions [15-17]. In addition, exosomes are also involved in angiogenesis, metastasis of tumor cells and transformation of normal cells into tumor cells [18]. Many types of cells such as mesenchymal cells, immune cells, and tumor cells may induce the release of exosomes, and increase in these cells indicate that exosomes are involved in tumorigenesis, development, metastasis, immune escape, and drug resistance [19]. Moreover, tumor-derived exosomes contain a large number of cancer-related serological markers, such as miRNAs, which could be used for detecting early HCC [20]. Despite several breakthroughs in the field of exosomes, the concrete biological role of exosomes has not been fully figured out. In this review, the source, structure, isolation of exosomes, and their impact and clinical application in HCC were described.

\section{The source and function of exosomes and the method for isolation of exosomes}

Exosomes were first discovered by Johnstone RM et al. in 1983 in mature sheep reticulocytes and named them as exosomes. They were initially perceived as cellular "debris" [21]. In 1996, Raposo et al. have confirmed the significant role of exosomes in antigen presentation from $\mathrm{B}$ cells, causing $\mathrm{T}$ cells responses [22]. So, the exosomes have gradually drawn the attention of several researchers, and is now considered to play an important role in the diagnosis and therapy of tumors. Exosomes are small phospholipid bilayer membrane nanovesicles that are formed by segregation of intracellular poly vesicles with cell membranes, followed by releasing into the extracellular space [23]. The detailed formation of exosomes is presented as follows. To begin with, the inward budding of cell membrane confirms the early endosomal stage [24], followed by the generation of multivesicular bodies (MVBs) by further inward budding of early endosomes and several miRNAs, proteins and other selected substances [25]. Finally, the MVBs either fuse with cell membrane, leading to the inclusion of extracellular DNA [26, 27], or fuse with lysosome, inducing the degradation of biological information containers in MVBs [28]. The endosomal sorting complex required for transport (ESCRT) mainly guides special molecules into the exosomes of MVBs, and is regarded as an important mechanism of synthesis [29, 30]. The ESCRT mainly contains4 core ingredients (ESCRT 0, I, II, and III), wherein the primary function is to provide ubiquitinated proteins to induce lysosomal degradation and protein reusing [31]. In the above process, ALG2-interacting protein $\mathrm{X}$ (ALIX), an accessory protein, plays an important role in interacting with ESCRT-III subunit SNF7, and then combines with MVB [32]. There are other mechanisms discovered by the researchers and are considered as ESCRT-independent, as they play a big role in this procedure [24]. Due to less understanding onESCRT-independent mechanisms, a great effort should be made to outline the detailed role of ESCRT-independent mechanism. The concrete mechanism of the formation of exosomes is clearly presented in Fig. 1.

Exosomes can be synthesized by any cells, such as B lymphocytes, $\mathrm{T}$ cells, mast cells, dendritic cells (DC), etc. and might be secreted to enter into other cells to carry out their function [34, 35]. Exosomes contain biologically active substances including proteins, RNA, DNA, cholesterol, ngosine, and so on, which range in size from 50 to $140 \mathrm{~nm}$ [36-38]. The density of exosomes is about $1.13 \sim 1.19 \mathrm{~g} / \mathrm{ml}$ [39]. Exosomes are abundant in human body, and can be found in biological fluids including urine, tears, plasma, breast milk, and cell culture supernatants [40-42]. Emerging evidence indicates that tumors during growth processing can secret exosomes. For example, Yang $L$ illustrated a new function of long noncoding ribonucleic acid (lncRNA HOTAIR) that induces MVBs for transporting them to plasma membrane, further propelling the release of exosomes from HCC [43].

The isolation of exosomes mainly involves 5 methods, which include differential ultracentrifugation [44, 45], polyethylene glycol (PEG) precipitation [46, 47], sucrose and iodixanol density ultracentrifugation [38], immunoaffinity (IAC) capture [48], and size exclusion chromatography $[49,50]$. In this review, we mainly introduced differential ultracentrifugation, as it is considered as gold standard for isolating exosomes [51]. Firstly, centrifugation at $300 \mathrm{~g}$ for $10 \mathrm{~min}$ is used to remove cellular debris. Secondly, the microvesicles were removed by centrifugation at $10,000 \mathrm{~g}$ for $30 \mathrm{~min}$. Thirdly, ultracentrifugation is performed at $1,00,000 \mathrm{~g}$, for $90 \mathrm{~min}$ at $4{ }^{\circ} \mathrm{C}$. Fourthly, the supernatant is discarded, followed by the addition of exosomal pellet to $1 \times \mathrm{PBS}$, and then washing it by ultracentrifugation at $1,00,000 \mathrm{~g}$ for $90 \mathrm{~min}$ at $4{ }^{\circ} \mathrm{C}$. Finally, the exosomes are resuspended in $500 \mu \mathrm{l} 1 \times \mathrm{PBS}$ and stored at $-80^{\circ} \mathrm{C}$ for further use [52]. But all these methods have their own limitations, and so further research of a more efficient method for isolating exosomes is needed.

\section{The role of exosomes in the process of chronic hepatitis B to hepatocellular carcinoma}

Several normal liver cells (such as hepatocytes,stellate cells and immune cells) can secrete exosomes, and meanwhile, these extracellular vesicles mediate normal communication between liver cells, maintaining liver 


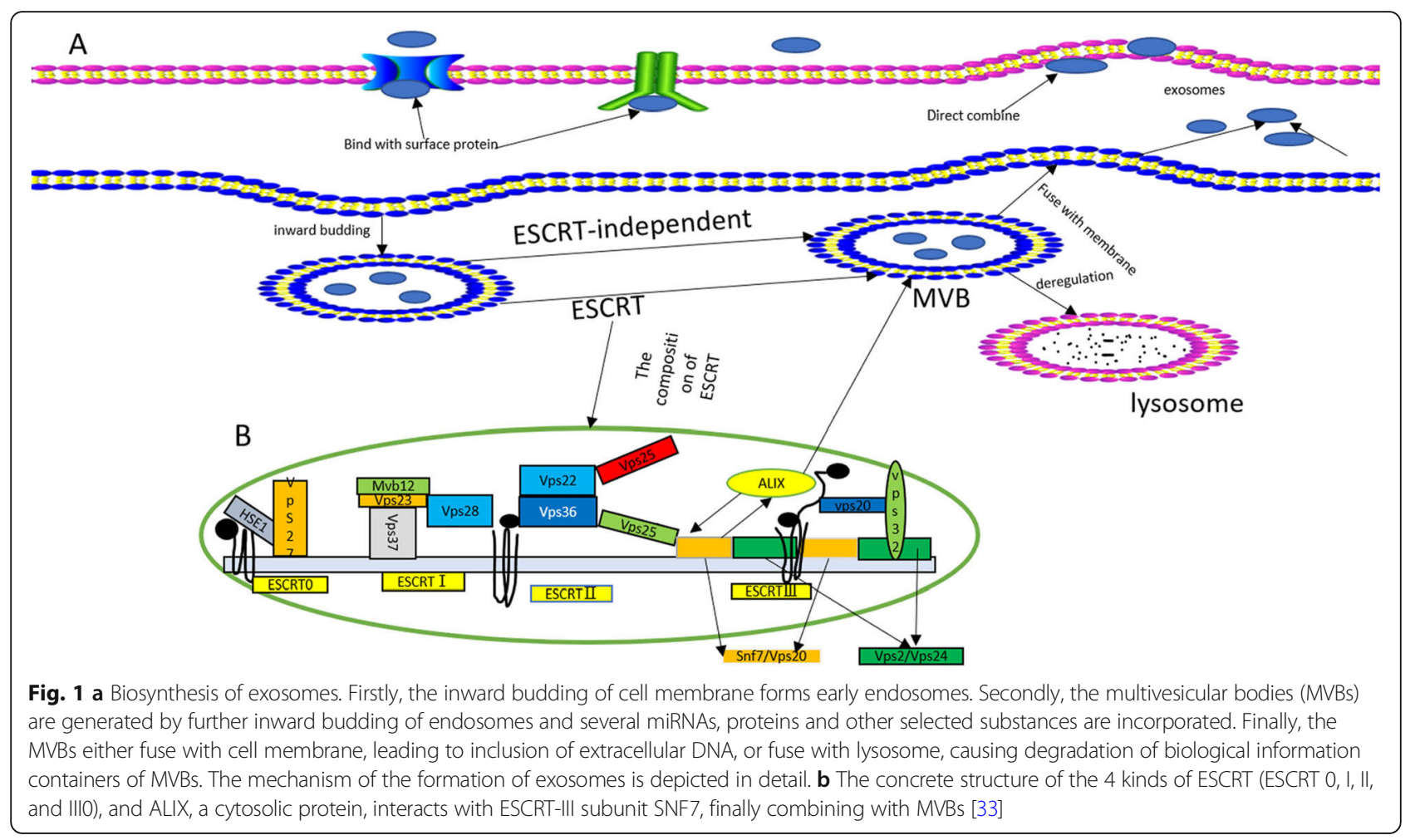

homeostasis [53]. According to Liu WH et al., the amount of serous exosomes in the cirrhotic stage, early HCC stage and late HCC stage is significantly higher than normal liver,liver degeneration and liver fibrosis, indicating that exosomes have high capability in the early detection of HCC [54]. Emerging evidence indicates that exosomes participate in the spread, immune regulation and antiviral response when the cells are infected by the viruses [55]. In china, the main reason for the cause of $\mathrm{HCC}$ is $\mathrm{CHB}$, and recent study by Kouwaki $\mathrm{T}$ et al. demonstrated that the infected virus in HCC induces exosomal miRNA-21 and miRNA-29, inhibiting macrophages and dendritic cells and releasing IL-12. It is widely accepted that IL-12 activates natural killer (NK) cells, thereby undermining the innate immune responses [56]. This may in turn cause chronic infection of hepatitis B (Fig. 2a), and long history of CHB may bring the effect of Hepatic fibrosis. It is evident that the hepatic stellate cells (HSCs) transdifferentiates into myofibroblasts in response to certain stimuli, and this is associated with the pathogenesis of hepatic fibrosis and the development of HCC [57]. According to the research proposed by Angeles Duran et al.,p62/SQSTM1, a negative regulator of liver inflammation and fibrosis, promotes Vitamin D receptor (VDR) signaling activation in HSCs, and mediates retinoid $\mathrm{X}$ receptor,which in turn promotes heterodimerization during the inhibition of liver inflammation and fibrosis [58]. The above processes make a great difference in recruiting the target gene. Also, loss of p62 expression in HSCs increases myofibroblastic differentiation, while suppresses fibrosis and inflammation via VDR agonists in chemically-induced murine fibrosis and tumor models. Carcinoma-associated fibroblasts (CAFs) are most frequently observed in human carcinomas, and involve alpha-smooth muscle actin-positive myofibroblasts and actin-negative fibroblasts that promote the growth and progression of tumor [59]. Although the critical role of p62/SQSTM1 and CAFs in liver inflammation, fibrosis and tumor progressionhas been discovered previously, research on p62/SQSTM and CAFs in exosomes is still lacking. It is widely accepted that exosomes are used as carriers to block several signal transduction pathways [60], and hence much attention has been paid on the relationship between p62/SQSTM, CAFs and exosomes in order to explore whether they could prevent liver inflammation, fibrosis and tumor progressionthrough blocking signal transduction pathways. Chen $\mathrm{L}$ et al. have discovered that the exosomes can utilize miR-214 to interplay cellular shuttling in order to regulate the formation of connective tissue growth factor 2 (CCN2) [61]. Overexpression of CCN2 by exosomes in HSCs is found in the activation process of liver fibrosis [62, 63] (Fig. 2b). After several years, hepatic fibrosis proliferates into HCC. Emergingevidence proves that exosomes play a bigger role in this stage. The lipid components of exosomes not only participate in a variety of $\mathrm{HCC}$ biological processes, but also protect tumor-derived exosomes from enzymatic degradation 


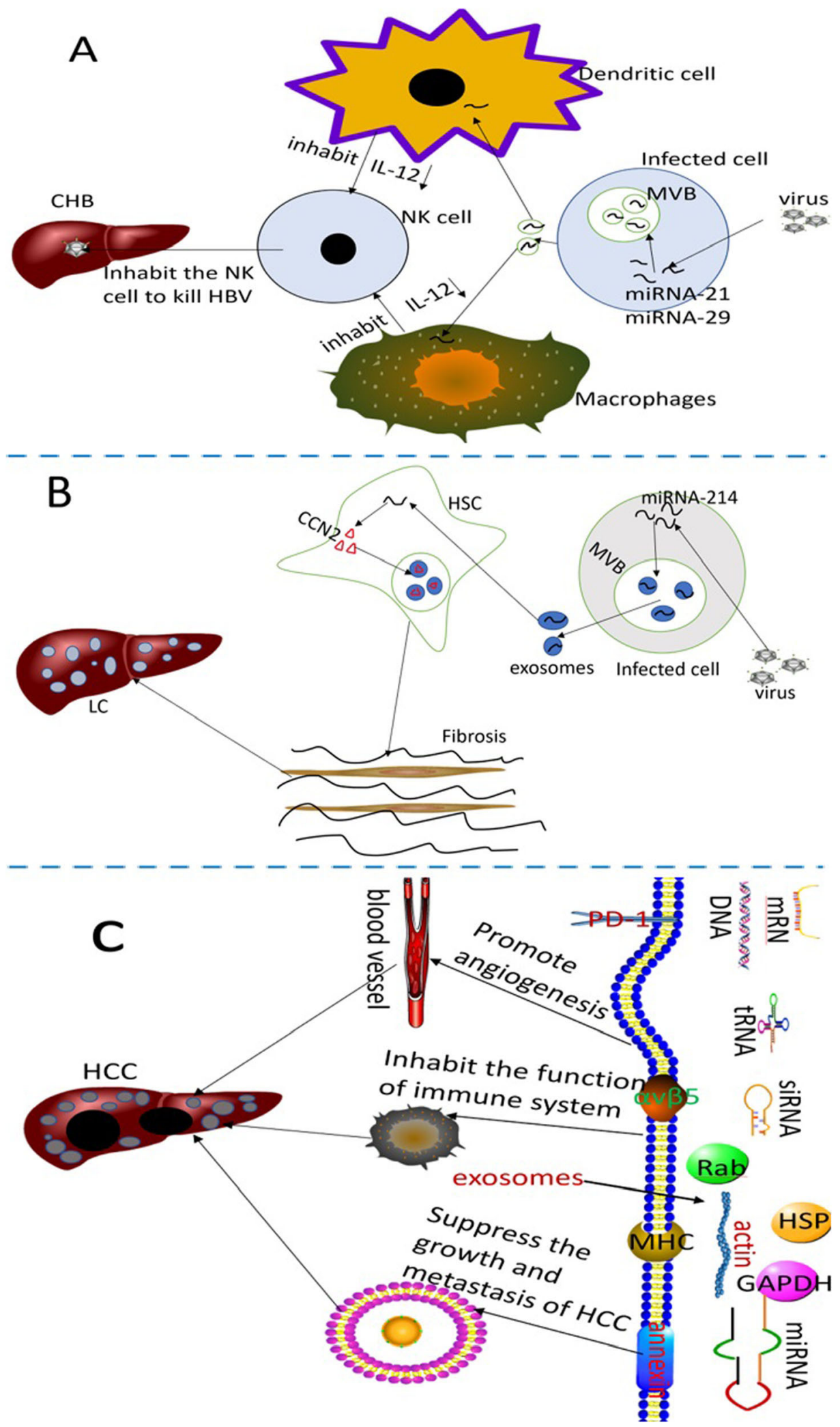

Fig. 2 The detailed mechanism of the role exosomes played in the process of CHB to HCC. a After infection with HBV, the viruses using exosomes secrete some substances that lead to chronic infection with HBV. b During the chronic infection with HBV, the viruses stimulate all kinds of cells to release exosomes, leading to liver cirrhosis (LC). c The concrete mechanism of exosomes during the formation of HCC 
[64]. The abundant protein Rab GTPase, annexin and similar exosomes could be used for HCC membrane transportation and fusion $[65,66]$. Heat shock proteins such as HSP70, HSP60 and HSP90 could be used for early diagnosis and treatment of liver cancer [67]. Exosomal proteomics indicate that exosomal integrin $\alpha \mathrm{v} \beta 5$ showed close association with liver metastasis [68] (Fig. 2c). Tumor susceptibility gene 101 protein (TSG101), major histocompatibility complex (MHC) molecules and the ESCRT-III binding protein ALIX might be used as biomarkers for diagnosis [69, 70]. In addition to this, many nucleic acids, including messenger RNA (mRNA), microRNAs (miRNAs), long noncoding ribonucleic acid (LncRNA) and DNA, regulate cell genetics as well as epigenetics [71]. The process of CHB to HCC is displayed vividly in Fig. 2.

\section{The relationship between exosomes and hepatocellular carcinoma}

Emerging evidence indicates that exosomes play a significant role in the formation and metastasis of $\mathrm{HCC}$ and the substances in exosomes are also used for clinical diagnosis and treatment. There are many types of liver cells, and so the intercellular communication is indispensable for liver cells to regulate and associate with each other [72]. Exosomes are novel research hot spots that play a critical role in intercellular communication [73]. In the next part, a thorough introduction on how the exosomes exert their role in HCC and how clinical exosomes are used as diagnostic and therapeutic tools is discussed.

\section{Exosomes regulate the growth and metastasis of HCC}

Increasing evidence reveals that exosomes could participate in the growth and metastasis of HCC, such as by secreting miRNAs or other substances by regulating the growth and metastasis of HCC. The major reason for difficult treatment and poor prognosis is due to intrahepatic and distal metastasis [4], and so it is imperative to make clear the detailed mechanism of HCC. HCC cells secrete miRNA-21, resulting in the activation of PDK1/ AKT signaling in HSCs. This in turn transforms HSC cells into CAFs. Activated CAFs secrete cytokines such as vascular endothelial growth factor (VEGF), matrix metalloprotein 2(MMP2), MMP9, bFGF and TGF- $\beta$ to further promote cancer development [74]. In addition, exosomes containing miRNA-21 and miR-29a bind to toll-like receptor (TLR) in immune cells, activate NF- $\mathrm{kB}$ pathway in TLR, secrete a series of inflammatory factors, and promote tumor growth and transfer [75]. Jinxing W et al. found that HCC-derived exosomes promoted growth, metastasis and invasion of tumor cells in a coculture experiment. In transporting the miRNAs to the recipient cell, Vps4A acts as an important negative regulator of exosomes. Through small RNA sequencing, Vps4A could regulate growth and metastasis of hepatoma cells by controlling the secretion and uptake of miRNAs [76]. Fang $\mathrm{T}$ et al. found that highly metastatic HCC cells produce exosomes containing miR-1247-3p, causing activation of $\beta 1$-integrin-NF- $\mathrm{KB}$ signaling pathway by CAFs. CAF further promotes the development of cancer by activating IL- 6 and IL- 8 . It can be seen that elevated exosomes of serum miR-1247-3p could promote lung metastasis in HCC patients [77]. Li B and other researchers have first discovered in an experiment that IncRNA FAL1 was upregulated in HCC exosomes, and meanwhile it could transfer to other HCC cells for promoting growth and migration of HCC [78]. Except HCC cells, other cells also can secret exosomes to promote HCC growth and reduce DNA damage. For example, Zhang $\mathrm{H}$ et al. in his research demonstrated that adipocytes could release exosomal circular RNAs (circRNAs) by reducing miR-34a and activating USP7/Cyclin A2 signaling pathway in tumor growth and reduce DNA injury [79].

\section{The role of exosomes in microcirculation of liver cancer Effect of exosomes on hepatocellular carcinoma angiogenesis}

Tumor growth requires blood vessels to provide a variety of nutrients. HCC is a highly angiogenic cancer, and VEGF plays a great role during the disease process. A recent study showed that mesenchymal stem cell (MSC)derived exosomes serve as an important mediator of intercellular communication and inhibit tumor angiogenesis by down-regulating VEGF [80]. Xue Jia Lin et al. found that miR-210 present in exosomes, which is secreted by HCC cells, could be transferred to endothelial cells to promote tumor angiogenesis by targeting SMAD4 and STAT6 [81]. Hiroshi Yukawa et al. found that HepG2-exosomes express NKG2D(an activated receptor of immune cells), and HSP70 (a stress-related heat shock protein). Both these act on VEGF receptors, leading to angiogenesis [82]. Fang $\mathrm{JH}$ et al. found that miR-103 in exosomes secreted by HCC increase vascular permeability and promote liver cancer metastasis by acting on endothelial cells [83]. Lee HYand other researchers have found that the expression of EIF3C could enhance HCC cells to secrete exosomes, causing tube formation of HUVEC cells and tumor growth, eventually leading to tumor angiogenesis [84]. Although HCC is a kind of high-vascular solid tumor, hypoxia also plays an important role in the formation of this cancer [85]. Thus, the two major problems that need to be solved include inhibition of angiogenesis and alleviation of hypoxia. Research regarding the role of exosomes under hypoxic conditions, and also the mechanism about angiogenesis is limited. Therefore, more efforts should 
be made on these two aspects for promoting further research on HCC.

\section{Exosomes participate in liver cancer epithelial-mesenchymal transition (EMT)}

Epithelial-mesenchymal transition (EMT) refers to the loss of polarity of epithelial cells and disruption of connections between cells, thereby transforming into cells with an interstitial phenotype [86]. EMT is classified into two types, complete EMT and partial EMT. Complete EMT is observed in individuals with vital role of it in metastasis initiation. Karaosmanoğlu Oand their group demonstrated reduction of E-cadherin and upregulation of ZEB2 in complete EMT, and meanwhile partial EMT involves increase of E-cadherin and decrease of vimentin and ZEB2 [87]. It is worth notifying that the EMT might causean increasein the cancer stem-like cells (CSCs), which refers to the highly tumorigenic subpopulation of tumor cells that exists at the top of the hierarchical tumor cell society $[88,89]$, leading to tumor heterogeneity and therapeutic resistance [89]. Emerging evidence showes that EMT can lead to tumor migration and metastasis [90]. Chen L et al. found that MHCC97Hderived exosomes could initiate EMT of HLE and Hep3B cells via MAPK/ERK signaling pathway. By downregulating Rab27a, excretion of MHCC97H-derived exosomes could be reduced, and EMT of parental MHCC97H cells could also be promoted [91]. Another research by Wang $\mathrm{C}$ et al. indicated that $\mathrm{Wnt} / \beta$-catenin signaling pathway miRNA25 was initially activated, and miRNA then directly inhibits Rho GDP dissociation inhibitor alpha (RhoGDI1). Reduction of RhoGDI1 could lead to upward expression of snail, eventually causing EMT [92]. In relation to this, Tang et al. have put forwarded an in vitro experiment that promotes cell proliferation, migration, and invasion in vitro by CTNND1(delta-catenin), and promotes HCC cell tumor formation and metastasis by CTNND1in vivo. According to a more thorough assay, CTNND1 indirectly enhanced $\mathrm{Wnt} / \beta$-catenin signaling to promote HCC metastasis. They also discovered that the expression of CTNND1showed a strong association when compared with $\beta$-catenin, WNT11, Cyclin D1, and BMP7 expressions in human HCC specimens. Knockdown of CTNND1 expression led to mesenchymal-epithelial transition (MET),but overexpression of it caused EMT and increased the potential of HCC metastasis [93, 94]. Studies on this topic are limited, but many evidences have proved that the functional molecules carried by tumorassociated exosomespromoted mesenchymal-associated gene expression and further induced EMT [95, 96]. So, it is necessary to perform a deeper exploration in the process of EMT.

\section{The role of exosomes in the immune regulation of liver} cancer

In recent years, the use of immune cells that target tumors has become a research hotspot. In a recent study conducted by $\mathrm{Lu} \mathrm{Z}$ et al., AFP-rich exosomes elicited a specific antitumor immune response, providing a new vaccine-free approach for treating HCC [97]. Rao Q also reported that exosomes derived from $\mathrm{HCC}$ antigens could elicit a stronger immune response than cell lysates. When using mouse cellderived exosomes for HCC treatment, the tumor immune microenvironment showed significant improvement, such as increased levels of T cells and $\gamma$-IFN, decreased levels of IL10 and TGF- $\beta$. We concluded that tumor cell-derived exosomes (TEX) that are expressed by HCC antigens might trigger a strong immune response in $\mathrm{DC}$, thereby improving the immune microenvironment of HCC [98]. Liu J et al. found that endoplasmic reticulum stress could trigger the release of exosomes, and upregulate the expression of PD-1 molecules in macrophages in liver cancer cells. The exosome miR-23a-PTEN-AKT pathway was activated, which then inhibited the function of $\mathrm{T}$ cells [99]. It is widely accepted that macrophages play a significant role in innate immune response, and mainly the classical (M1) macrophages lead to anti-tumor activity. But alternate (M2) macrophages mainly promote tumorigenesis and tumor growth $[100,101]$. A comparison experiment by Xue Liet al. demonstrated that knockdown of IncRNA UC339 in THP-1 cells led to M1 increase, and overexpression of IncRNA TUC339 in THP-1 cells caused M2 increase [102].

\section{Use of exosomes in the diagnosis and prognosis of liver cancer}

Due to spatial heterogeneity and temporal heterogeneity of tumor, traditional method of tissue specimen biopsy cannot obtain the full and dynamic information of tumor tissues. In recent years, a novel diagnostic technology named "liquid biopsy" has been emerged to overcome the shortcomings of traditional tissue biopsy [103, 104]. In the protection of phospholipid bilayer membrane, the exosomal substances cannot be degraded by any enzyme, and so exosomes are considered as an appropriate diagnostic tool.

As the exosomes have many unique substances that can be expressed by tumor cells, we utilized them for the early detection of tumor. miRNAs are a class of conserved RNAs in the evolutionary history of humans and also participate in the development of liver cancer. According to the latest research, miRNA is considered as a potentially new and ideal marker [105]. Won Sohn et al. found that the levels of miR-18a, miR-221, miR-222 and miR-224 in exosomes of patients with HCC were lower than those in patients with $\mathrm{HBV}$, indicating that these 3 miRNAs could be used as novel serum markers for detecting HCC [106]. Apart from miRNAs, IncRNAs are 
also used as biomarkers for clinical diagnosis of HCC [107]. Xiang Ma et al. discovered in an experiment that exosomes mediate the regulation of lncRNAs X-inactivespecific transcript in the expression of blood cells, and indicate that Xist expressed by mononuclear cells and granulocytes might act as valuable biomarkers in the diagnosis of female HCC patients [108].

Another study also reported that miR-30d, miR-140 and miR-29b showed significance in the survival of patients with liver cancer. Therefore, these exosomal miRNAs act as prognostic biomarkers for liver cancer and guide in the treatment of advanced liver cancer [109]. For proving the relationship of circulating exosomal noncoding RNAs (ncRNAs) in tumors, Lee YRet al. have conducted a series of experiments, which eventually showesa relationship of ncRNAs (miRNA21 and lncRNA-ATB) with TNM staging and prognosis of HCC [110]. Xu Het al. indicates that serum exosomallncRNAs ENSG00000258332.1 and LINC00635 combined with serum AFP might be a promising method for diagnosis and prognosis of HCC [111]. Other experiment also supported that exosomal lncRNA acts as a prognostic factor in HCC, and lncRNA (LINC00161) significantly upregulates in HCC patients when compared to normal patients, which is well stabilized and specific [112]. In addition to lncRNA, circPTGR1, a circRNA, is particularly expressed in exosomes of $97 \mathrm{~L}$ and LM3 cells, and is increased in the serum exosomes of $\mathrm{HCC}$ patients. Wang $\mathrm{G}$ and his collaborator indicated that it could be used for clinical staging and prognosis [113].

\section{Application of exosomes in the treatment of liver cancer}

HCC is not a sensitive disease to common chemotherapy. miR-122 could promote the sensitivity of HCC cells to chemotherapeutic drugs, and exosomes can be used as a biological carrier of miRNAs. These findings indicates that exosomes from self-body cells have less immunogenicity when compared with other vehicles [114]. Emerging evidenceshows the feasibility of applying exosomes as nanocarriers, for example, low immunogenicity, high biocompatibility, less toxicity and so on $[115,116]$. At the same time, MSCs could secrete large amounts of exosomes. Researchers have found that transfection of miRNA-122 into adipose derived mesenchymal stem cells formed by MSCs could produce exocrine bodies containing miRNA-122, improving the miR-122-target gene

Table 1 The function of the substance in HCC exosomes

\begin{tabular}{|c|c|c|c|c|}
\hline Components & Functions & First author/s & Year & References \\
\hline Rab protein, GTPase, annexin & Membrane transport and fusion & CORDONNIER M & 2017 & {$[34]$} \\
\hline \multicolumn{5}{|l|}{ miRNA } \\
\hline miR-1247-3p & Promote lung migration of liver cancer & Fang $T$ & 2018 & {$[77]$} \\
\hline miRNA-210 & Promotes angiogenesis & $\operatorname{Lin} X J$ & 2018 & [81] \\
\hline miR-103 & Vascular permeability and metastasis & Fang JH & 2018 & {$[83]$} \\
\hline miR-23a-3p & Inhibits the function of T-cell & Liu J & 2019 & [99] \\
\hline miR-122 & Improve the treatment effect & Lou G & 2015 & {$[117]$} \\
\hline miR-335 & Novel therapeutic strategy & Wang F & 2018 & [118] \\
\hline microRNA-25-5p & Migration、 Invasion & Liu $\mathrm{H}$ & 2018 & [122] \\
\hline miR-320a-PBX3 & Proliferation, metastasis & Zhang Z & 2017 & [123] \\
\hline miR-718 & Prediction the prognosis of $\mathrm{HCC}$ & Sugimachi K & 2015 & [124] \\
\hline miR-665 & Biomarker & Qu Z & 2017 & {$[125]$} \\
\hline \multicolumn{5}{|l|}{ HSP } \\
\hline HSP70, HSP60 and HSP90 & Diagnosis and treatment & CORDONNIER M & 2017 & {$[34]$} \\
\hline InCRNA HOTAIR & The release of exosomes & YANG L & 2019 & [45] \\
\hline RNA and DNA & Regulation cell genetics and epigenetics & ZHANG $X$ & 2015 & [71] \\
\hline Vps4A & Tumor suppressor & Jin-xing Wei & 2015 & [76] \\
\hline \multicolumn{5}{|l|}{ IncRNA } \\
\hline IncRNA-ATB & Novel prognosis of biomarker and therapeutic targets & Lee YR & 2019 & {$[110]$} \\
\hline LUCAT1 and CASC9 & Biomarker & Gramantieri L & 2018 & [126] \\
\hline \multicolumn{5}{|l|}{ circRNA } \\
\hline circ-DB & Promote HCC growth and reduce DNA damage & Zhang H & 2019 & [79] \\
\hline circPTGR1 & Clinical stage and prognosis & Wang G & 2019 & [113] \\
\hline
\end{tabular}

HCC hepatocellular carcinoma, miR/miRNA micro ribonucleic acid, HSP heat shock proteins, circRNA Circular RNAs, IncRNA Long noncoding ribonucleic acid 
expression and promoting sensitivity of cancer cells to chemotherapy [117]. Fang Wang and other researchers have found that stellate cell-derived EVs could load miR335-5p. What makes us exhilarated is that miR-335-5 could be introduced into HCC cells to inhibit tumor growth and metastasis, which thereby provides a new treatment strategy for liver cancer [118]. Another research by Kenji Takahashiet al. have found that during the process of mediating chemotherapeutic stress response, RNAi-mediated knockdown of EVs (exosomes) lncRNAs could reduce the function and progression of tumor cells in HCC, which promotes the treatment of HCC [119].

\section{Conclusion and future prospects}

Exosomes are involved in the occurrence, development, and metastasis of tumors, providing new clues for the treatment of HCC. We also found that many substances in exosomes including miRNAs serve as new biomarkers. It is also of great significance in improving the early diagnosis of HCC. Therefore, exosomes have become a hot research topic currently. This review has first introduced the role of exosomes in the development of CHB to HCC. Secondly, the mechanism of exosomes in tumor growth and metastasis is also discussed. The last but not the least, it is elucidated that exosomes could be used for clinical diagnosis and treatment. The function of the substance in HCC exosomes are conclued (Table 1). Although several studies have been put forwarded in investigating the relationship of exosomes and liver cancer, research on the formation mechanism of liver cancer by exosomes is still not deep enough. This is because of effective separation and specific detection of circulating exosomes in cancer cells [24, 125]. Meanwhile, the use of exosomes in studying the 4 serum markers of liver cancer (AFP, AFP-L3, GP73, and GPC3) has rarely been reported. Different researchers have drawn different views regarding the same exosomes. The major reason for this might be due to individual differences. So, environment, aging, gender, reason for HCC, and multi-center should be combined to produce more accurate results. For over the past several years, exosomes are used in immune therapeutic method only in 3 phase I clinical trials [126]. With more research conducted on exosomes, it is believed that exosomes could be successfully used in clinical diagnosis of early stage $\mathrm{HCC}$ in near future.

\footnotetext{
Abbreviations

ALIX: ALG2-interacting protein X; CAFs: Carcinoma-associated fibroblasts; CCN2: Connective tissue growth factor 2; CHB: Chronic hepatitis B; circRNAs: circular RNAs; CSCs: Cancer stem-like cells; EMT: Epithelialmesenchymal transition; ESCRT: Endosomal sorting complex required for transport; HCC: Hepatocellular carcinoma; HSCs: Hepatic stellate cells; HSP: Heat shock proteins; IAC: Immunoaffinity; LC: Liver cirrhosis; IncRNA: Long noncoding ribouncleic acid; MHC: Major histocompatibility complex; MMP: Matrix metalloprotein; MSC: Mesenchymal stem cell;
}

MSCs: Mesenchymal cells; MVB: Multivesicular bodies; NK: Natural killer; PEG: Polyethylene glycol; Rho GDI: Rho GDP dissociation inhibitor alpha; TEX: Tumor cell-derived exosomes; TLR: Toll-like receptor; TSG101: Tumor susceptibility gene 101 protein; VEGF: Vascular endothelial growth factor

\section{Acknowledgments \\ Not applicable}

\section{Authors' contributions}

LWH, LX and LCY conceived of the presented idea. LN, CK and WM researched on the background of the study. CDX, JFF and ZLP critically reviewed the manuscript. All authors contributed to and approved the final manuscript.

\section{Funding}

This study was funded by the National Natural Science Foundation of China (81603552); Beijing Science and Technology Fund of Traditional Chinese Medicine (JJ2018-32); Beijing Fengtai Health System Research Project(201863); Youan Foundation of Liver Disease and AIDS (YNKTTS20180123); The Natural Science Foundation of Beijing (7172103); Beijing Engineering Research Center for Precision Medicine and Transformation of Hepatitis and Liver Cancer (BG0320).

Availability of data and materials Not applicable

Ethics approval and consent to participate Not applicable

\section{Consent for publication}

Not applicable

\section{Competing interests}

The authors declare that they have no competing interests.

\section{Author details}

${ }^{1}$ Beijing Youan Hospital, Capital Medical University, Beijing, China. ${ }^{2}$ Beijing Institute of Hepatology, Beijing Youan Hospital, Capital Medical University, 8 Xitoutiao, Youanmenwai,Fengtai District, Beijing 100069, China. ${ }^{3}$ Department of Maternity, Yanan University Affiliated Hospital, Yanan, China.

Received: 25 July 2019 Accepted: 4 October 2019

Published online: 04 January 2020

References

1. Torre LA, Bray F, Siegel RL, et al. Global cancer statistics, 2012. CA Cancer J Clin. 2015:65(2):87-108.

2. Qu Z, Wu J, Wu J, et al. Exosomes derived from HCC cells induce sorafenib resistance in hepatocellular carcinoma both in vivo and in vitro. J Exp Clin Cancer Res. 2016;35(1):159.

3. Grohmann M, Wiede F, Dodd GT, et al. Obesity Drives STAT-1-Dependent NASH and STAT-3-Dependent HCC. Cell. 2018;175(5):1289-1306.e1220.

4. Chen $R, X u X$, Tao $Y$, et al. Exosomes in hepatocellular carcinoma: a new horizon. Cell Commun Signal. 2019;17(1):1.

5. Zheng C, Zheng L, Yoo JK, et al. Landscape of Infiltrating T Cells in Liver Cancer Revealed by Single-Cell Sequencing. Cell. 2017;169(7):1342-1356. e1316.

6. Zhang ZF, Feng XS, Chen $\mathrm{H}$, et al. Prognostic significance of synergistic hexokinase-2 and beta2-adrenergic receptor expression in human hepatocelluar carcinoma after curative resection. BMC Gastroenterol. 2016; 16(1):57.

7. Cainap C, Qin S, Huang WT, et al. Linifanib versus Sorafenib in patients with advanced hepatocellular carcinoma: results of a randomized phase III trial. J Clin Oncol. 2015;33(2):172-9.

8. Peng $\mathrm{S}$, Zhao $\mathrm{Y}, \mathrm{Xu}$ F, et al. An updated meta-analysis of randomized controlled trials assessing the effect of sorafenib in advanced hepatocellular carcinoma. PLoS One. 2014;9(12):e112530.

9. El-Serag HB, Rudolph KL. Hepatocellular carcinoma: epidemiology and molecular carcinogenesis. Gastroenterology. 2007:132(7):2557-76.

10. El-Serag HB. Hepatocellular carcinoma. N Engl J Med. 2011;365(12):1118-27. 
11. Llovet JM, Ricci S, Mazzaferro V, et al. Sorafenib in advanced hepatocellular carcinoma. N Engl J Med. 2008;359(4):378-90.

12. Roma-Rodrigues C, Raposo LR, Cabral R, et al. Tumor Microenvironment Modulation via Gold Nanoparticles Targeting Malicious Exosomes: Implications for Cancer Diagnostics and Therapy. Int J Mol Sci. 2017;18(1).

13. Chen G, Huang AC, Zhang W, et al. Exosomal PD-L1 contributes to immunosuppression and is associated with anti-PD-1 response. Nature. 2018;560(7718):382-6.

14. Gougelet A. Exosomal microRNAs as a potential therapeutic strategy in hepatocellular carcinoma. World J Hepatol. 2018;10(11):785-9.

15. Kalra H, Simpson RJ, Ji H, et al. Vesiclepedia: a compendium for extracellular vesicles with continuous community annotation. PLoS Biol. 2012;10(12): e1001450.

16. Kim DK, Kang B, Kim OY, et al. EVpedia: an integrated database of highthroughput data for systemic analyses of extracellular vesicles. J Extracell Vesicles. 2013;2.

17. Mathivanan S, Fahner CJ, Reid GE, et al. ExoCarta 2012: database of exosomal proteins, RNA and lipids. Nucleic Acids Res. 2012;40(Database issue):D1241-4.

18. Guo W, Gao Y, Li N, et al. Exosomes: New players in cancer (Review). Oncol Rep. 2017;38(2):665-75.

19. Sun F, Wang JZ, Luo JJ, et al. Exosomes in the Oncobiology, Diagnosis, and Therapy of Hepatic Carcinoma: A New Player of an Old Game. Biomed Res Int. 2018;2018:2747461.

20. Melo SA, Sugimoto H, O'Connell JT, et al. Cancer exosomes perform cellindependent microRNA biogenesis and promote tumorigenesis. Cancer Cell. 2014;26(5):707-21.

21. Johnstone RM, Adam M, Hammond JR, et al. Vesicle formation during reticulocyte maturation. Association of plasma membrane activities with released vesicles (exosomes). J Biol Chem. 1987;262(19): 9412-20.

22. Raposo G, Nijman HW, Stoorvogel W, et al. B lymphocytes secrete antigenpresenting vesicles. J Exp Med. 1996;183(3):1161-72.

23. Raab-Traub N, Dittmer DP. Viral effects on the content and function of extracellular vesicles. Nat Rev Microbiol. 2017;15(9):559-72.

24. Shao $\mathrm{H}, \mathrm{Im}$ H, Castro $\mathrm{CM}$, et al. New Technologies for Analysis of Extracellular Vesicles. Chem Rev. 2018;118(4):1917-50.

25. Piper RC, Katzmann DJ. Biogenesis and function of multivesicular bodies Annu Rev Cell Dev Biol. 2007;23:519-47.

26. Colombo M, Raposo G, Thery C. Biogenesis, secretion, and intercellular interactions of exosomes and other extracellular vesicles. Annu Rev Cell Dev Biol. 2014;30:255-89.

27. Thery C. Exosomes: secreted vesicles and intercellular communications. F1000 Biol Rep. 2011;3:15.

28. Luzio JP, Gray SR, Bright NA. Endosome-lysosome fusion. Biochem Soc Trans. 2010;38(6):1413-6.

29. Hurley $\mathrm{JH}$, Hanson PI. Membrane budding and scission by the ESCRT machinery: it's all in the neck. Nat Rev Mol Cell Biol. 2010;11(8):556-66.

30. Henne WM, Buchkovich NJ, Emr SD. The ESCRT pathway. Dev Cell. 2011; 21(1):77-91.

31. Wollert T, Hurley JH. Molecular mechanism of multivesicular body biogenesis by ESCRT complexes. Nature. 2010;464(7290):864-9.

32. Cardona-Lopez X, Cuyas L, Marin E, et al. ESCRT-III-Associated Protein ALIX Mediates High-Affinity Phosphate Transporter Trafficking to Maintain Phosphate Homeostasis in Arabidopsis. Plant Cell. 2015;27(9): 2560-81.

33. Bowers $\mathrm{K}$, Lottridge J, Helliwell SB, et al. Protein-protein interactions of ESCRT complexes in the yeast Saccharomyces cerevisiae. Traffic. 2004;5(3):194-210.

34. Kogure T, Lin WL, Yan IK, et al. Intercellular nanovesicle-mediated microRNA transfer: a mechanism of environmental modulation of hepatocellular cancer cell growth. Hepatology. 2011;54(4):1237-48.

35. Kamerkar S, Lebleu VS, Sugimoto $H$, et al. Exosomes facilitate therapeutic targeting of oncogenic KRAS in pancreatic cancer. Nature. 2017;546(7659): 498-503.

36. Wang Y, Balaji V, Kaniyappan S, et al. The release and trans-synaptic transmission of Tau via exosomes. Mol Neurodegener. 2017;12(1):5.

37. Chivet $M$, Javalet $C$, Hemming $F$, et al. Exosomes as a novel way of interneuronal communication. Biochem Soc Trans. 2013;41(1):241-4.

38. Kowal J, Arras G, Colombo M, et al. Proteomic comparison defines novel markers to characterize heterogeneous populations of extracellular vesicle subtypes. Proc Natl Acad Sci U S A. 2016;113(8):E968-77.
39. Merchant ML, Rood IM, Deegens JKJ, et al. Isolation and characterization of urinary extracellular vesicles: implications for biomarker discovery. Nat Rev Nephrol. 2017;13(12):731-49.

40. Yu S, Cao H, Shen B, et al. Tumor-derived exosomes in cancer progression and treatment failure. Oncotarget. 2015;6(35):37151-68.

41. Wang $H$, Hou L, Li A, et al. Expression of serum exosomal microRNA-21 in human hepatocellular carcinoma. Biomed Res Int. 2014;2014:864894.

42. Oosthuyzen $W$, Sime NE, IVy JR, et al. Quantification of human urinary exosomes by nanoparticle tracking analysis. J Physiol. 2013;591(23):5833-42.

43. Yang L, Peng X, Li Y, et al. Long non-coding RNA HOTAIR promotes exosome secretion by regulating RAB35 and SNAP23 in hepatocellular carcinoma. Mol Cancer. 2019;18(1):78.

44. Andre F, Schartz NE, Movassagh M, et al. Malignant effusions and immunogenic tumour-derived exosomes. Lancet. 2002;360(9329):295-305.

45. Cai S, Luo B, Jiang P, et al. Immuno-modified superparamagnetic nanoparticles via host-guest interactions for high-purity capture and mild release of exosomes. Nanoscale. 2018;10(29):14280-9.

46. Yamamoto KR, Alberts BM, Benzinger R, et al. Rapid bacteriophage sedimentation in the presence of polyethylene glycol and its application to large-scale virus purification. Virology. 1970;40(3):734-44.

47. Weng Y, Sui Z, Shan Y, et al. Effective isolation of exosomes with polyethylene glycol from cell culture supernatant for in-depth proteome profiling. Analyst. 2016;141(15):4640-6.

48. Greening DW, Xu R, Ji H, et al. A protocol for exosome isolation and characterization: evaluation of ultracentrifugation, density-gradient separation, and immunoaffinity capture methods. Methods Mol Biol. 2015; 1295:179-209.

49. Hong CS, Funk $S$, Muller $L$, et al. Isolation of biologically active and morphologically intact exosomes from plasma of patients with cancer. J Extracell Vesicles. 2016;5:29289.

50. Vickers KC, Palmisano BT, Shoucri BM, et al. MicroRNAs are transported in plasma and delivered to recipient cells by high-density lipoproteins. Nat Cell Biol. 2011;13(4):423-33.

51. Thery C, Amigorena S, Raposo G, et al. Isolation and characterization of exosomes from cell culture supernatants and biological fluids. Curr Protoc Cell Biol. 2006; Chapter 3: Unit 322.

52. GUPTA S, RAWAT S, ARORA V, et al. An improvised one-step sucrose cushion ultracentrifugation method for exosome isolation from culture supernatants of mesenchymal stem cells. Stem Cell Res Ther. 2018;9(1):180.

53. Santangelo L, Battistelli C, Montaldo C, et al. Functional Roles and Therapeutic Applications of Exosomes in Hepatocellular Carcinoma. Biomed Res Int. 2017;2017:2931813.

54. Liu WH, Ren LN, Wang X, et al. Combination of exosomes and circulating microRNAs may serve as a promising tumor marker complementary to alpha-fetoprotein for early-stage hepatocellular carcinoma diagnosis in rats. J Cancer Res Clin Oncol. 2015;141(10):1767-78.

55. Chahar HS, Bao X, Casola A. Exosomes and Their Role in the Life Cycle and Pathogenesis of RNA Viruses. Viruses. 2015;7(6):3204-25.

56. Kouwaki T, Okamoto M, Tsukamoto H, et al. Extracellular Vesicles Deliver Host and Virus RNA and Regulate Innate Immune Response. Int J Mol Sci. 2017;18(3).

57. Devhare PB, Ray RB. Extracellular vesicles: Novel mediator for cell to cell communications in liver pathogenesis. Mol Asp Med. 2018;60:115-22.

58. Duran A, Hernandez ED, Reina-Campos M, et al. p62/SQSTM1 by Binding to Vitamin D Receptor Inhibits Hepatic Stellate Cell Activity, Fibrosis, and Liver Cancer. Cancer Cell. 2016;30(4):595-609.

59. Yoshida GJ, Azuma A, Miura Y, et al. Activated Fibroblast Program Orchestrates Tumor Initiation and Progression; Molecular Mechanisms and the Associated Therapeutic Strategies. Int J Mol Sci. 2019;20(9).

60. Gangoda L, Boukouris S, Liem M, et al. Extracellular vesicles including exosomes are mediators of signal transduction: are they protective or pathogenic? Proteomics. 2015;15(2-3):260-71.

61. Chen $L$, Charrier $A$, Zhou $Y$, et al. Epigenetic regulation of connective tissue growth factor by MicroRNA-214 delivery in exosomes from mouse or human hepatic stellate cells. Hepatology. 2014;59(3):1118-29.

62. Huang G, Brigstock DR. Regulation of hepatic stellate cells by connective tissue growth factor. Front Biosci (Landmark Ed). 2012;17:2495-507.

63. Charrier A, Chen $\mathrm{R}$, Chen $\mathrm{L}$, et al. Exosomes mediate intercellular transfer of pro-fibrogenic connective tissue growth factor (CCN2) between hepatic stellate cells, the principal fibrotic cells in the liver. Surgery. 2014;156(3):548-55. 
64. Wu Z, Zeng Q, Cao K, et al. Exosomes: small vesicles with big roles in hepatocellular carcinoma. Oncotarget. 2016;7(37):60687-97.

65. Raulf N, Lucarelli P, Thavaraj S, et al. Annexin A1 regulates EGFR activity and alters EGFR-containing tumour-derived exosomes in head and neck cancers. Eur J Cancer. 2018;102:52-68.

66. Blanc L, Vidal M. New insights into the function of Rab GTPases in the context of exosomal secretion. Small GTPases. 2018;9(1-2):95-106.

67. Cordonnier M, Chanteloup $\mathrm{G}$, Isambert $\mathrm{N}$, et al. Exosomes in cancer theranostic: Diamonds in the rough. Cell Adhes Migr. 2017;11(2):151-63.

68. Hoshino A, Costa-Silva B, Shen TL, et al. Tumour exosome integrins determine organotropic metastasis. Nature. 2015;527(7578):329-35.

69. Budnik V, Ruiz-Canada C, Wendler F. Extracellular vesicles round off communication in the nervous system. Nat Rev Neurosci. 2016;17(3):160-72.

70. Kowal J, Tkach M, Thery C. Biogenesis and secretion of exosomes. Curr Opin Cell Biol. 2014;29:116-25.

71. Zhang $X$, Yuan $X$, Shi $H$, et al. Exosomes in cancer: small particle, big player. J Hematol Oncol. 2015;8:83.

72. Crispe IN. The liver as a lymphoid organ. Annu Rev Immunol. 2009;27:147-63.

73. Luga $V$, Zhang L, Viloria-Petit AM, et al. Exosomes mediate stromal mobilization of autocrine Wnt-PCP signaling in breast cancer cell migration. Cell. 2012;151(7):1542-56.

74. Zhou Y, Ren H, Dai B, et al. Hepatocellular carcinoma-derived exosomal miRNA-21 contributes to tumor progression by converting hepatocyte stellate cells to cancer-associated fibroblasts. J Exp Clin Cancer Res. 2018, 37(1):324.

75. Yu X, Odenthal M, Fries JW. Exosomes as miRNA Carriers: FormationFunction-Future. Int J Mol Sci. 2016;17(12)

76. Wei JX, Lv LH, Wan $Y L$, et al. Vps4A functions as a tumor suppressor by regulating the secretion and uptake of exosomal microRNAs in human hepatoma cells. Hepatology. 2015;61(4):1284-94.

77. FANG T, LV H, LV G, et al. Tumor-derived exosomal miR-1247-3p induces cancer-associated fibroblast activation to foster lung metastasis of liver cancer. Nat Commun. 2018;9(1):191

78. Li B, Mao R, Liu C, et al. LncRNA FAL1 promotes cell proliferation and migration by acting as a CeRNA of miR-1236 in hepatocellular carcinoma cells. Life Sci. 2018;197:122-9.

79. ZHANG H, DENG T, Ge $\mathrm{S}$, et al. Exosome circRNA secreted from adipocytes promotes the growth of hepatocellular carcinoma by targeting deubiquitination-related USP7. Oncogene. 2019;38(15):2844-59.

80. Lee JK, Park SR, Jung BK, et al. Exosomes derived from mesenchymal stem cells suppress angiogenesis by down-regulating VEGF expression in breast cancer cells. PLoS One. 2013;8(12):e84256.

81. Lin XJ, Fang JH, Yang XJ, et al. Hepatocellular Carcinoma Cell-Secreted Exosomal MicroRNA-210 Promotes Angiogenesis In Vitro and In Vivo. Mol Ther Nucleic Acids. 2018;11:243-52.

82. Yukawa $H$, Suzuki $K$, Aoki $K$, et al. Imaging of angiogenesis of human umbilical vein endothelial cells by uptake of exosomes secreted from hepatocellular carcinoma cells. Sci Rep. 2018;8(1):6765.

83. Fang $J H$, Zhang ZJ, Shang LR, et al. Hepatoma cell-secreted exosomal microRNA-103 increases vascular permeability and promotes metastasis by targeting junction proteins. Hepatology. 2018;68(4):1459-75.

84. Lee HY, Chen CK, Ho CM, et al. EIF3C-enhanced exosome secretion promotes angiogenesis and tumorigenesis of human hepatocellular carcinoma. Oncotarget. 2018;9(17):13193-205.

85. Wu XZ, Xie GR, Chen D, et al. J Gastroenterol Hepatol. 2007;22(8):1178-82.

86. Blackwell RH, Foreman KE, Gupta GN. The Role of Cancer-Derived Exosomes in Tumorigenicity \& Epithelial-to-Mesenchymal Transition. Cancers (Basel). 2017;9(8).

87. Karaosmanoglu $\mathrm{O}$, Banerjee $\mathrm{S}$, Sivas $\mathrm{H}$. Identification of biomarkers associated with partial epithelial to mesenchymal transition in the secretome of slug over-expressing hepatocellular carcinoma cells. Cell Oncol (Dordr). 2018;41(4):439-53.

88. Kalluri R, Weinberg RA. The basics of epithelial-mesenchymal transition. J Clin Invest. 2009;119(6):1420-8.

89. Yoshida GJ, Saya H. Therapeutic strategies targeting cancer stem cells. Cancer Sci. 2016;107(1):5-11.

90. Vella LJ. The emerging role of exosomes in epithelial-mesenchymaltransition in cancer. Front Oncol. 2014;4:361.

91. Chen L, Guo P, He Y, et al. HCC-derived exosomes elicit HCC progression and recurrence by epithelial-mesenchymal transition through MAPK/ERK signalling pathway. Cell Death Dis. 2018;9(5):513.
92. Wang C, Wang X, Su Z, et al. MiR-25 promotes hepatocellular carcinoma cell growth, migration and invasion by inhibiting RhoGDI1. Oncotarget. 2015; 6(34):36231-44.

93. Tang B, Tang F, Wang Z, et al. Overexpression of CTNND1 in hepatocellular carcinoma promotes carcinous characters through activation of Wnt/betacatenin signaling. J Exp Clin Cancer Res. 2016;35(1):82.

94. Yoshida GJ. Emerging role of epithelial-mesenchymal transition in hepatic cancer. J Exp Clin Cancer Res. 2016;35(1):141.

95. Syn N, Wang L, Sethi G, et al. Exosome-Mediated Metastasis: From EpithelialMesenchymal Transition to Escape from Immunosurveillance. Trends Pharmacol Sci. 2016;37(7):606-17.

96. Greening DW, Gopal SK, Mathias RA, et al. Emerging roles of exosomes during epithelial-mesenchymal transition and cancer progression. Semin Cell Dev Biol. 2015;40:60-71.

97. Lu Z, Zuo B, Jing R, et al. Dendritic cell-derived exosomes elicit tumor regression in autochthonous hepatocellular carcinoma mouse models. J Hepatol. 2017;67(4):739-48.

98. RAO Q, ZUO B, LU Z, et al. Tumor-derived exosomes elicit tumor suppression in murine hepatocellular carcinoma models and humans in vitro. Hepatology. 2016;64(2):456-72.

99. Liu J, Fan L, Yu H, et al. Endoplasmic Reticulum Stress Promotes Liver Cancer Cells to Release Exosomal miR-23a-3p and Up-regulate PD-L1 Expression in Macrophages. Hepatology. 2019.

100. Zhou D, Huang C, Lin Z, et al. Macrophage polarization and function with emphasis on the evolving roles of coordinated regulation of cellular signaling pathways. Cell Signal. 2014;26(2):192-7.

101. Ivashkiv LB. Epigenetic regulation of macrophage polarization and function. Trends Immunol. 2013;34(5):216-23.

102. Li X, Lei Y, Wu M, et al. Regulation of Macrophage Activation and Polarization by HCC-Derived Exosomal IncRNA TUC339. Int J Mol Sci. 2018;19(10).

103. Alix-Panabieres C, Pantel K. Clinical Applications of Circulating Tumor Cells and Circulating Tumor DNA as Liquid Biopsy. Cancer Discov. 2016;6(5):479-91.

104. Diaz LA Jr, Bardelli A. Liquid biopsies: genotyping circulating tumor DNA. J Clin Oncol. 2014;32(6):579-86.

105. Zhang YC, Xu Z, Zhang TF, et al. Circulating microRNAs as diagnostic and prognostic tools for hepatocellular carcinoma. World J Gastroenterol. 2015; 21(34):9853-62.

106. Sohn W, Kim J, Kang SH, et al. Serum exosomal microRNAs as novel biomarkers for hepatocellular carcinoma. Exp Mol Med. 2015;47:e184.

107. Tang J, Jiang R, Deng L, et al. Circulation long non-coding RNAs act as biomarkers for predicting tumorigenesis and metastasis in hepatocellular carcinoma. Oncotarget. 2015;6(6):4505-15.

108. Ma X, Yuan T, Yang C, et al. X-inactive-specific transcript of peripheral blood cells is regulated by exosomal Jpx and acts as a biomarker for female patients with hepatocellular carcinoma. Ther Adv Med Oncol. 2017;9(11):665-77.

109. Yu LX, Zhang BL, Yang $Y$, et al. Exosomal microRNAs as potential biomarkers for cancer cell migration and prognosis in hepatocellular carcinoma patientderived cell models. Oncol Rep. 2019;41(1):257-69.

110. Lee YR, Kim G, Tak WY, et al. Circulating exosomal noncoding RNAs as prognostic biomarkers in human hepatocellular carcinoma. Int J Cancer. 2019;144(6):1444-52.

111. Xu H, Chen $Y$, Dong $X$, et al. Serum Exosomal Long Noncoding RNAs ENSG00000258332.1 and LINC00635 for the Diagnosis and Prognosis of Hepatocellular Carcinoma. Cancer Epidemiol Biomark Prev. 2018;27(6):710-6.

112. Sun L, Su Y, Liu X, et al. Serum and exosome long non coding RNAs as potential biomarkers for hepatocellular carcinoma. J Cancer. 2018;9(15):2631-9.

113. Wang G, Liu W, Zou Y, et al. Three isoforms of exosomal circPTGR1 promote hepatocellular carcinoma metastasis via the miR449a-MET pathway. EBioMedicine. 2019:40:432-45.

114. Clayton A, Harris CL, Court J, et al. Antigen-presenting cell exosomes are protected from complement-mediated lysis by expression of CD55 and CD59. Eur J Immunol. 2003;33(2):522-31.

115. Syn NL, Wang L, Chow EK, et al. Exosomes in Cancer Nanomedicine and Immunotherapy: Prospects and Challenges. Trends Biotechnol. 2017; 35(7):665-76.

116. Ha D, Yang N, Nadithe V. Exosomes as therapeutic drug carriers and delivery vehicles across biological membranes: current perspectives and future challenges. Acta Pharm Sin B. 2016;6(4):287-96.

117. Lou G, Song X, Yang F, et al. Exosomes derived from miR-122-modified adipose tissue-derived MSCs increase chemosensitivity of hepatocellular carcinoma. J Hematol Oncol. 2015;8:122. 
118. Wang F, Li L, Piontek K, et al. Exosome miR-335 as a novel therapeutic strategy in hepatocellular carcinoma. Hepatology. 2018;67(3):940-54.

119. Takahashi K, Yan IK, Wood J, et al. Involvement of extracellular vesicle long noncoding RNA (linc-VLDLR) in tumor cell responses to chemotherapy. Mol Cancer Res. 2014;12(10):1377-87.

120. Liu H, Chen W, Zhi X, et al. Tumor-derived exosomes promote tumor selfseeding in hepatocellular carcinoma by transferring miRNA-25-5p to enhance cell motility. Oncogene. 2018;37(36):4964-78.

121. Zhang Z, Li X, Sun W, et al. Loss of exosomal miR-320a from cancerassociated fibroblasts contributes to HCC proliferation and metastasis. Cancer Lett. 2017;397:33-42.

122. Sugimachi $K$, Matsumura T, Hirata $H$, et al. Identification of a bona fide microRNA biomarker in serum exosomes that predicts hepatocellular carcinoma recurrence after liver transplantation. Br J Cancer. 2015;112(3):532-8.

123. Qu Z, Wu J, Wu J, et al. Exosomal miR-665 as a novel minimally invasive biomarker for hepatocellular carcinoma diagnosis and prognosis. Oncotarget. 2017;8(46):80666-78.

124. Gramantieri L, Baglioni M, Fornari F, et al. LncRNAs as novel players in hepatocellular carcinoma recurrence. Oncotarget. 2018;9(80):35085-99.

125. Melo SA, Luecke LB, Kahlert C, et al. Glypican-1 identifies cancer exosomes and detects early pancreatic cancer. Nature. 2015;523(7559):177-82.

126. Gilligan KE, Dwyer RM. Engineering Exosomes for Cancer Therapy. Int J Mol Sci. 2017;18(6).

\section{Publisher's Note}

Springer Nature remains neutral with regard to jurisdictional claims in published maps and institutional affiliations.

Ready to submit your research? Choose BMC and benefit from:

- fast, convenient online submission

- thorough peer review by experienced researchers in your field

- rapid publication on acceptance

- support for research data, including large and complex data types

- gold Open Access which fosters wider collaboration and increased citations

- maximum visibility for your research: over $100 \mathrm{M}$ website views per year

At BMC, research is always in progress.

Learn more biomedcentral.com/submissions 